\title{
Use of the age-adjusted D-dimer as a screening tool for pulmonary thromboembolic disease in an older inpatient population
}

Authors: Lauren Pickering, Joanna Davison and Vikas Bhalla

\section{Aims}

Does an age-adjusted D-dimer threshold (age multiplied by ten) improve the specificity without modifying the sensitivity when compared to the absolute $\mathrm{D}$-dimer value in an older inpatient population?

\section{Methods}

All patients that had undergone a CT pulmonary angiogram (CTPA) between 1 August 2012 and 1 August 2013 were collated. We calculated and compared the sensitivity and specificity of the D-dimer test as a screening tool for a pulmonary embolus using both the absolute value (greater than $250 \mathrm{ng} / \mathrm{mL}$ in our trust) versus an age-adjusted $\mathrm{D}$-dimer value (age multiplied by ten).

A comparison was then made in two separate populations: those aged from 17 to 69 years of age and those aged from 70 to 100 years of age. Patients that had undergone a CTPA but without a D-dimer were excluded.

\section{Results}

613 patients had undergone a CTPA, of which 334 were female and 279 male. The median age was 79 years. There were 308 patients in the 17-69 age group, with 305 patients in the 70-100 age group.

The table below illustrates the sensitivities and specificities in the two different age categories.

\begin{tabular}{lll} 
& \multicolumn{2}{c}{ Age (years) } \\
\cline { 2 - 3 } & $\mathbf{1 7 - 6 9}$ & $\mathbf{7 0 - 1 0 0}$ \\
Sensitivity of absolute D-dimer value & $94.4 \%$ & $95.2 \%$ \\
Sensitivity of age-adjusted D-dimer value & $90.1 \%$ & $91 \%$ \\
Specificity of absolute D-dimer value & $24 \%$ & $8.3 \%$ \\
Specificity of age-adjusted D-dimer value & $59.1 \%$ & $67.1 \%$
\end{tabular}

\section{Conclusions}

D-dimer is a fibrin degradation product; however, concentrations increase with age. Older patients undergo unnecessary investigations when an absolute D-dimer value is used as a screening tool, as opposed to an age-adjusted D-dimer value. We have shown that, in a population aged 70 or over, the age-adjusted D-dimer value improves the specificity without modifying the sensitivity in screening for pulmonary thromboembolic disease. For our older patients, perhaps the age-adjusted D-dimer should be used routinely as a screening tool for pulmonary thromboembolic disease? 